\title{
(6) Schistosoma mansoni antigen Sm-p80: OPEN ACCESS prophylactic efficacy using TLR4 agonist vaccine adjuvant glucopyranosyl lipid A-Alum in murine and non-human primate models
}

\author{
Weidong Zhang, ${ }^{1,2}$ Gul Ahmad, ${ }_{1}^{3}$ Adebayo J Molehin, ${ }^{1,2}$ Workineh Torben, ${ }_{1}^{4}$ \\ Loc Le, ${ }^{5}$ Eunjee Kim, ${ }^{1,2}$ Samra Lazarus, ${ }^{1,2}$ Arif J Siddiqui, ${ }^{1,2}$ Darrick Carter, ${ }^{6}$ \\ Afzal A Siddiqui ${ }^{1,2}$
}

Additional material is published online only. To view please visit the journal online (http://dx.doi.org/10. 1136/jim-2018-000786).

For numbered affiliations see end of article.

\section{Correspondence to} Dr Afzal A Siddiqui, Department of Internal Medicine 3601 4th Street, Mail Stop 9410, Texas Tech University Health Sciences Center, Lubbock, TX 79430 9410 Tel: (806) 743-2638; Fax (806) 743-4030 ; Afzal.Siddiqui@ttuhsc.edu

WZ and GA contributed equally.

Accepted 3 June 2018 Published Online First 10 July 2018
Check for updates

To cite: Zhang $\mathrm{W}_{\text {, }}$ Ahmad G, Molehin AJ, et al. J Investig Med 2018;66:1124-1132.

\section{ABSTRACT}

Sm-p80, the large subunit of Schistosoma mansoni calpain, is a leading candidate for a schistosomiasis vaccine. The prophylactic and antifecundity efficacy of $\mathrm{Sm}$-p80 has been tested in three animal models (mouse, hamster and baboon) using a multitude of vaccine formulations and approaches. In our continual effort to enhance the vaccine efficacy, in this study, we have utilized the adjuvant, synthetic hexa-acylated lipid A derivative, glucopyranosyl lipid A (GLA) formulated in aluminum (GLA-Alum) with recombinant Sm-p80. The rSm-p80+GLA-Alum immunization regimen provided $33.33 \%-53.13 \%$ reduction in worm burden in the mouse model and $38 \%$ worm burden reduction in vaccinated baboons. Robust Sm-p80-specific immunoglobulin $(\mathrm{lg}) \mathrm{G}$, $\lg G 1, \lg G 2 a$ and $\lg M$ responses were observed in all immunized animals. The rSm-p80+GLA-Alum coadministration induced a mix of T-helper (Th) cells (Th1, Th2 and Th17) responses as determined via the release of interleukin (IL)-2, IL-4, IL-18, IL-21, IL-22 and interferon- $\gamma$.

\section{INTRODUCTION}

Schistosomiasis is a neglected tropical disease of significant public health importance that has the potential to impact up to an estimated one billion people worldwide. Approximately 237 million people are currently infected and an additional 800 million people are in danger of being exposed to this parasitic infection in 78 countries. ${ }^{1-3}$ The estimated disability adjusted life years associated with schistosomiasis is 3.6 million, ${ }^{4}$ and health-related quality of life indicate that schistosomiasis is potentially causing a much higher disease burden than was previously estimated. ${ }^{5}$ Mass antiparasitic drug administration programs using praziquantel and other control strategies have made inroads in reducing the disease burden of schistosomiasis. ${ }^{6}$ However, most experts agree that a sustainable and meaningful reduction in the disease burden and transmission would only be

\section{Significance of this study}

What is already known about this subject?

- Schistosomiasis is a neglected tropical parasitic disease affecting over 230 million people worldwide.

- Current control programs centered on mass drug administration of praziquantel are inadequate.

- Elimination of schistosomiasis is only attainable through integrated control programs with an effective vaccine serving as a fulcrum.

- The large subunit of Schistosoma mansoni calcium-activated neutral protease (calpain), Sm-p80, is a leading schistosomiasis vaccine.

What are the new findings?

- Sm-p80 vaccine formulated in synthetic hexa-acylated lipid A derivative, glucopyranosyl lipid A (GLA) formulated in aluminum (GLA-Alum) provided significant protection against $S$. mansoni infections in mice and baboons.

- GLA adjuvant formulated in Alum did not maximize the immunogenicity and efficacy of $\mathrm{Sm}$-p80 vaccine.

- Immunizations with Sm-p80+GLA-Alum induced significant production of Sm-p80specific antibodies (immunoglobulin (Ig)G, $\lg \mathrm{G} 1, \lg \mathrm{G} 2 \mathrm{a}$ and $\lg \mathrm{M})$.

- Sm-p80-mediated balanced T-helper (Th) cells (Th1/Th2/Th17) immune responses are associated with immune protection in vaccinated animals.

possible through the deployment of a vaccine in conjunction with current control programs. ${ }^{47}$

The large subunit of Schistosoma mansoni calpain, Sm-p80, is expressed in all S. mansoni life cycle stages. ${ }^{8-10} \mathrm{Sm}-\mathrm{p} 80$ plays critical roles in schistosome tegument biogenesis/renewal, a mechanism employed to modulate and/or 


\section{Significance of this study}

How might these results change the focus of research or clinical practice?

- The results from this pilot study reinforce the continued development of $\mathrm{Sm}$-p80 as a schistosomiasis vaccine. Due to its inefficiency at overriding the parasiteinduced Th2 responses, GLA-Alum adjuvant may not be suitable for the next phase of schistosomiasis vaccine development.

evade their host immune attack. ${ }^{8}{ }^{11}$ With respect to schistosomiasis vaccine development, $\mathrm{Sm}-\mathrm{p} 80$ is regarded as a leading candidate having demonstrated significant protection against $S$. mansoni challenge infections in addition to cross-species protection against Schistosoma haematobium and Schistosoma japonicum infections in rodent and baboon models. ${ }^{1212-16}$

Adjuvants are utilized to improve or modulate the intrinsic immunogenicity of an antigen to selectively switch the onset of a specific cell-mediated response in addition to the antibody response. ${ }^{1718}$ Based on our previous efficacy studies using Sm-p80 antigen formulated in either glucopyranosyl lipid A in stable, oil-in-water emulsion (GLA-SE) ${ }^{19}$ or alum hydroxide ${ }^{16}$ which showed significant protection against $S$. mansoni infections, we hypothesized that $S \mathrm{~m}-\mathrm{p} 80$ vaccine formulated with GLA adsorbed on alum hydroxide (GLA-Alum) would enhance its efficacy. GLA and Alum hydroxide adjuvants are currently used in many commercially available vaccine formulations and are potent stimulators of T-helper (Th) 1 and Th2 cell immune responses, respectively. ${ }^{21}$ In this present study, we assessed the protective efficacy of Sm-p80+GLA-Alum vaccine against S. mansoni infections in mice as well as in a pilot study using non-human primate model of infection and disease.

\section{MATERIALS AND METHODS}

\section{Animals and parasites}

Mice aged 3-4week, female C57BL/6, were purchased from Charles River Laboratories International (Wilmington, Massachusetts, USA). Animal husbandry and all performed procedures were guided by the principles of the Institutional Animal Care and Use Committee. Olive baboons (Papio anubis) aged 11-16 years were bred in the Association for Assessment and Accreditation of Laboratory Animal Care and International-accredited facilities at the University of Oklahoma Health Sciences Center (OUHSC). Animals were prescreened for intestinal and blood parasites and for antibodies that are cross-reactive to Sm-p 80 . Infected Biomphalaria glabrata snails, the intermediate host of $S$. mansoni, were provided by Schistosomiasis Resource Center (Biomedical Research Institute, Rockville, Maryland, USA). On the day of challenge, infected snails were exposed under light to induce cercarial shedding. The number and viability of cercariae were counted under a light microscope.

\section{Preparation of recombinant protein Sm-p80}

The recombinant $\mathrm{Sm}-\mathrm{p} 80$ protein $(\mathrm{rSm}-\mathrm{p} 80)$ was produced using prokaryotic expression system as previously described. ${ }^{10}$ In brief, the full length $S m-p 80$ gene cloned into pCold II (GenScript Corp., Piscataway, New Jersey, USA) and transformed into BL21 (DE3) Escherichia coli strain (Invitrogen Corp., Carlsbad, California, USA). The expression of recombinant protein was induced by $0.75 \mathrm{mM}$ isopropyl $\beta$-D-1-thiogalactopyranoside. Expressed protein was purified by affinity chromatography followed by size exclusion chromatography. Endotoxin levels in purified protein samples were analyzed with a Limulus amebocyte lysate assay (Charles River Laboratories International) and the quality of $\mathrm{rSm}-\mathrm{p} 80$ analyzed by sodium dodecyl sulfate polyacrylamide gel (SDS-PAGE) and western blotting.

\section{Animal immunization and challenge}

Formulated GLA-Alum was provided by PAI Life Sciences. For the mouse experiment, three independent vaccine trials were conducted with a total of 10 mice per trial, divided randomly into the control group $(\mathrm{n}=5)$ and the experimental group $(n=5)$. Each mouse in the control groups was immunized with $5 \mu \mathrm{g}$ GLA-Alum, while those in the experimental group were immunized with $25 \mu \mathrm{g}$ rSm-p 80 in combination with $5 \mu \mathrm{g}$ GLA-Alum. Primary immunization followed by two boosters were administered intramuscularly at weeks 0,4 and 8 , respectively. Four weeks after the last immunization of each trial, the mice were challenged with $150 \mathrm{~S}$. mansoni cercariae via tail exposure. For the pilot baboon experiment, six baboons were randomly divided into control $(n=3)$ and experimental $(n=3)$ groups. The control group received $50 \mu \mathrm{g}$ GLA-Alum, while the experimental group received $250 \mu \mathrm{g} \mathrm{rSm}$-p 80 with $50 \mu \mathrm{g}$ GLA-Alum at weeks 0,4 and 8 . Four weeks after last immunization, all the baboons were exposed to $1000 \mathrm{~S}$. mansoni cercariae at the deposit site of the axillary cavity. Online Supplementary table 1 shows immunization protocol and the experimental schedule.

\section{Animal necropsy, worm and egg burden}

All animals were sacrificed at week 6 (mouse experiment) or week 8 (baboon experiment) post challenge. Adult worms were recovered by perfusion of the portal system and the mesenteric veins. ${ }^{23}$ The liver and intestine of individual animals were excised for digestion overnight at $37^{\circ} \mathrm{C}$ in $4 \%$ potassium hydroxide. ${ }^{23}$ Eggs were counted to determine the egg burden for each animal. The reduction $(\mathrm{P})$ in worm and egg burdens were calculated by comparing control (C) and experimental (I) groups with a standard formula: $P \%=\frac{C-I}{C} \times 100 \% .^{12}$

\section{Serum antibody response to vaccination}

Blood samples were collected from individual animals at 2-week intervals (mouse experiment) or 4-week intervals (baboon experiment) and sera were isolated for antibody level determination using ELISA as already described. ${ }^{12}{ }^{15}$ Briefly, 96-well plates were coated with rSm-p80 $(1.2 \mu \mathrm{g} /$ well). Sm-p80-specific antibody titres for total IgG subtypes, IgA and $\operatorname{IgM}$ were determined using either horseradish peroxidase labeled antimouse or antimonkey secondary antibodies (Alpha Diagnostics International, San Antonio, Texas, USA). The results were expressed as mean of endpoint titres \pm SD as previously published. ${ }^{24}$ 
Cell proliferation and Th1/Th2 skewed cytokine response Peripheral blood mononuclear cells (PBMCs) from the two groups of baboons were isolated by density gradient centrifugation using HISTOPAQUE-1077 (Sigma-Aldrich, St. Louis, Missouri, USA) every 4 weeks. After euthanasia, spleen cells and lymph node cells from baboons were isolated and stored. In the mouse experiment, the spleens were removed from each animal after sacrifice and splenocyte suspensions were prepared for 3-(4,5-dimethylethiazol-2-yl)-2,5-diphenytetrazolium bromide (MTT) assay. A standard MTT assay was performed as follows: in a 96-well flat bottom plate, PBMCs, lymph node cells and splenocytes $\left(5 \times 10^{5} / 200 \mu \mathrm{L} /\right.$ well $)$ were stimulated with $0.5 \mu \mathrm{g}$ of concanavalin (ConA), $1.2 \mu \mathrm{g}$ of recombinant Sm-p80 or $1.2 \mu \mathrm{g}$ of ovalbumin (OVA). After 48 hours of incubation at $37^{\circ} \mathrm{C}, 100 \mu \mathrm{L}$ of supernatant was gathered for cytokine production assay by ELISA. Twenty microlitres of MTT was then added to the remainder during cell proliferation, as described in previous papers. ${ }^{13}{ }^{1525-29}$ Hundred microlitres of dimethyl sulfoxide was then loaded into each well to dissolve the formazan salt crystals. The plate was read at OD $500 \mathrm{~nm}$ and SI was calculated as the ratio of OD $550 \mathrm{~nm}$ of stimulated cells to non-stimulated cells. Th1/Th2 cytokines (interleukin (IL)-2, IL-4, interferon (IFN)- $\gamma$ and IL-10) were detected using a murine cytokine Th1/Th2 ELISA panel kit (ebiosciences, San Diego, California, USA) and monkey cytokine ELISA kits (U-cyTech, The Netherlands). These were performed according to the manual provided by the manufacturer. All in vitro experiments were done in triplicates.

\section{mRNA expressions of cytokines detected by reverse transcription-PCR (RT-PCR)}

In a six-well plate, $2 \times 10^{7}$ PBMCs and lymph node cells from baboons and splenocytes from mice and baboons were seeded and maintained in complete Roswell Park Memorial Institute (RPMI) 1640 medium containing $10 \%$ fetal bovine serum, $100 \mu \mathrm{g} / \mathrm{mL}$ of penicillin G, 100 $\mu \mathrm{g} / \mathrm{mL}$ of streptomycin and $10 \mu \mathrm{g} / \mathrm{mL}$ of gentamicin, with or without $12 \mu \mathrm{g} / \mathrm{mL}$ of recombinant Sm-p80 for 24 hour at $37^{\circ} \mathrm{C}$. After 24 hour, the cells were collected and washed with ice-cold phosphate buffered saline. RNA was extracted using the TRIzol method (Invitrogen Corp.). RNA $(0.5 \mu \mathrm{g})$ was used to synthesize cDNA by reverse transcription. Expression of cytokines (mouse: IL-1 $\alpha$, IL-1 $\beta$, IL-2, IL-3, IL-4, IL-5, IL-6, IL-7, MIP-2, IL-9, IL-10, IL-11, IL-12 $\alpha$, IL-12 $\beta$, IL-13, IL-14, IL-15, IL-16, IL-17, IL-18, IL-19, IL-20, IL-21，IL-22, IL-23, tumour necrosis factor (TNF)- $\alpha$, IFN- $\gamma$, transforming growth factor (TGF)- $\beta 1$ and TGF- $\beta 2$; baboon: IL-1 $\alpha$, IL-1 $\beta$, IL-2, IL-4, IL-5, IL-6, IL-8, IL-10, IL-12 $\alpha$, IL-12 $\beta$, IL-18, IL-21, IL-22, IL-23, TGF- $\beta 1$, TGF- $\beta 2$ and IFN- $\gamma$ and glyceraldehyde 3 -phosphate dehydrogenase (GAPDH) were determined via RT-PCR as outlined previously. ${ }^{12} 15162629$ The RT-PCR products were analyzed on a $2 \%$ agarose gel, and the relative difference was determined using Quantity One (V.4.6.2, Bio-Rad, Hercules, California, USA). All in vitro experiments were done in triplicates.
Th1, Th2 and Th17 phenotype analysis by Enzyme-Linked ImmunoSpot (ELISPOT)

ELISPOT assay was used to spot IL-4, IFN- $\gamma$ and IL-17 secreting cells stimulated with recombinant Sm-p80 in vitro. Briefly, PBMCs from baboons and splenocytes from mice were seeded $\left(3 \times 10^{5}\right.$ cells $/ 100 \mu \mathrm{L} /$ well $)$ on the 96-well precoated (anti-IFN- $\gamma$, anti-IL-4, or anti-IL-17, R\&D, Minneapolis, Minnesotta, USA; U-cyTech). The cells were stimulated with either $0.5 \mu \mathrm{g}$ ConA or $5 \mathrm{ng}$ phorbol 12-myristate 13 -acetate plus $100 \mathrm{ng}$ ionomycin or $1.2 \mu \mathrm{g}$ recombinant $\mathrm{Sm}$-p 80 or $1.2 \mu \mathrm{g}$ ovalbumin and incubated at $37^{\circ} \mathrm{C}$ with $5 \% \mathrm{CO}_{2}$ for 48 hours. Spotforming unit (SFU) representing single cells were counted using an ELISPOT Bioreader 5000 (ImmunoBioSystem, The Colony, Texas, USA). Antigen-specific SFU per well was calculated by subtracting its background value (medium control well without antigen), as described in previous studies. ${ }^{12-14} 1928$ All assays were done in triplicates.

\section{Flow cytometry}

In a 24 -well plate, $2 \times 10^{6}$ PBMCs from baboons and $4 \times 10^{6}$ splenocytes from mice were cultured in each well in the presence of $12 \mu \mathrm{g} / \mathrm{mL}$ of recombinant Sm-p80 for 24 hours at $37^{\circ} \mathrm{C}$. The cells were maintained solely in normal complete RPMI media as a control. BD GolgiStop Protein Transport Inhibitor was added in the last 10 hours of incubation. Cells maintained in phorbol 12-myristate 13 -acetate $(100 \mathrm{ng} / \mathrm{mL})$ and ionomycin $(1 \mu \mathrm{g} / \mathrm{mL})$ were processed as positive controls. The splenocyte staining procedure was performed following the instructions provided by the Mouse Th1/Th2/Th17 Phenotype Kit (BD Biosciences, San Diego, California, USA). Following two washes with staining buffer, the cells were fixed and permeabilized with cold BD Cytofix buffer and BD Perm/ Wash buffer. Twenty microlitres of cocktail antibody (antimouse CD4Peridinin Chlorophyll Protein Complex (PERCP)-CY5.5, antimouse IL-17A phycoerythrin (PE), antimouse IFN- $\gamma$ flourescein isothiocyanate (FITC) and antimouse IL-4allophycocyanin (APC) was added to each tube and incubated for $30 \mathrm{~min}$ at room temperature. For baboon PBMCs, PerCP-Cy5.5-conjugated mouse antihuman CD4 was used to identify CD4+ T cells. The addition of APC-mouse antihuman IL-4, PE-mouse antihuman IFN- $\gamma$ and FITC-mouse antihuman IL-17 were then added for intracellular staining to detect IFN- $\gamma$, IL-4 and IL-17 secreting cells. All immunological reagents and antibodies were purchased from BD Biosciences (San Diego, California, USA). Data were collected using CellQuset Prosoftware (BD Biosciences) and analyzed via FlowJo software (Tree Star, Ashland, Oregon, USA).

\section{Statistical analysis}

Significance between the control and experimental groups was calculated via the independent two-sample t-test or the Mann-Whitney-Wilcoxon rank-sum test, using Sigma Plot (V.11.0, Systat Software). Bonferroni adjustments were included for multiple comparisons to reduce the risk of reaching false conclusions based on chance. $\mathrm{P}<0.05$ was considered significant. 
Table 1 Cumulative parasitological data for all mouse trials

\begin{tabular}{|c|c|c|c|c|c|c|c|c|c|c|}
\hline \multirow[b]{2}{*}{ Vaccine group } & \multirow[b]{2}{*}{$\begin{array}{l}\text { Vaccine } \\
\text { trial }\end{array}$} & \multirow[b]{2}{*}{$\mathbf{n}$} & \multicolumn{2}{|l|}{ Worm burden } & \multicolumn{2}{|c|}{ Egg burden (per g) } & \multirow[b]{2}{*}{$\begin{array}{l}\text { Total egg burden in } \\
\text { tissue }\end{array}$} & \multicolumn{2}{|c|}{ Egg reduction } & \multirow[b]{2}{*}{$\begin{array}{l}\text { Gross tissue } \\
\text { egg reduction }\end{array}$} \\
\hline & & & Total worm & $\begin{array}{l}\text { Worm } \\
\text { protection }\end{array}$ & Liver & Intestine & & Liver & Intestine & \\
\hline \multirow[t]{3}{*}{ GLA-Alum } & Trial $1 \mathrm{C}$ & 5 & 133 & \multirow{3}{*}{$\begin{array}{l}43.37 \% \\
(p \leq 0.001)\end{array}$} & $1009.66 \pm 637.63$ & $310.53 \pm 173.99$ & $660.10 \pm 332.65$ & \multirow{3}{*}{$\begin{array}{l}\text { No } \\
\text { protection }\end{array}$} & \multirow[t]{3}{*}{$38.07 \%$} & \multirow{3}{*}{$5.57 \%$} \\
\hline & Trial $2 \mathrm{C}$ & 5 & 158 & & $1512.63 \pm 605.11$ & $207.02 \pm 69.84$ & $859.82 \pm 360.28$ & & & \\
\hline & Trial 3C & 5 & 124 & & $697.85 \pm 231.38$ & $314.16 \pm 87.21$ & $506.27 .00 \pm 132.96$ & & & \\
\hline \multicolumn{2}{|c|}{ Group mean $( \pm \mathrm{SE})$} & & $138.33 \pm 10.17$ & & $1073.38 \pm 294.52$ & $277.23 \pm 65.18$ & $675.31 \pm 165.61$ & & & \\
\hline \multirow{3}{*}{$\begin{array}{l}\text { rSm-p80+GLA- } \\
\text { Alum }\end{array}$} & Trial 1E & 5 & 90 & & $1781.48 \pm 117.07$ & $256.80 \pm 77.55$ & $1019.14 \pm 262.59$ & & & \\
\hline & Trial 2E & 5 & 76 & & $831.18 \pm 409.28$ & $181.37 \pm 74.87$ & $506.27 \pm 224.05$ & & & \\
\hline & Trial 3E & 5 & 69 & & $698.50 \pm 210.51$ & $76.89 \pm 32.38$ & $387.69 \pm 144.27$ & & & \\
\hline \multicolumn{2}{|c|}{ Group mean $( \pm \mathrm{SE})$} & & $78.33 \pm 6.17$ & & $1103.72 \pm 195.18$ & $171.68 \pm 39.94$ & $637.70 \pm 130.65$ & & & \\
\hline
\end{tabular}

GLA-Alum, glucopyranosyl lipid A (GLA) formulated in aluminum.

\section{RESULTS}

\section{Protective efficacy of rSm-p80 vaccination with GLA- Alum adjuvant}

The protective efficacy of Sm-p80+GLA-Alum was evaluated in both baboon and three independent mouse trials as described above. In mouse trial 1, the mean worm recovered from the GLA-Alum control group $(27 \pm 3.54)$ compared with the group immunized with Sm-p80+GLA-Alum $(18 \pm 0.84)$ revealed a $33.33 \%$ significant reduction in worm burden $(p=0.045)$ (online Supplementary table 2). In mouse trial 2 , we also observed a statistically significant reduction $(53.13 \%, \mathrm{p}=0.0048)$ in worm burden when the average worm numbers was compared between the control group (32 \pm 3.12$)$ and the experimental group $(15 \pm 2.89$ worms). We also observed a $44 \%$ reduction in worm burden in mouse trial $3(\mathrm{p}=0.075)$ (online Supplementary table 2). Overall, we observed a slight significant reduction in tissue egg load in all mouse trials except for the intestinal egg burden in trial $3(75.52 \%$ egg reduction, $p=0.032)$ (online Supplementary table 2). The overall cumulative parasitological data for all three independent mouse trials are shown in table 1 and online Supplementary figure 1B. For the pilot baboon trial, we observed a significant reduction (38.53\%) in worm burden when the average worm numbers of the GLA-Alum control group $(646.33 \pm 17.34)$ was compared with that of the Sm-p80+GLA-Alum immunized group $(397.33 \pm 40.05$ worms) (table 2 and online Supplementary figure $1 \mathrm{~A})$. We observed a $37.95 \%$ reduction in intestinal egg load in baboons immunized with Sm-p80+GLA-Alum when compared with the controls. However, there was no difference in hepatic egg burden between both groups (table 2). Statistically, no vaccine-mediated preferential killing of either male or female worm was observed. Specifically, the recovery of male and female worms cumulatively was as follows: control group ((mice) 65.94\% male, $34.05 \%$ female), vaccine group ((mice) $59.74 \%$ male, $40.25 \%$ female), control group ((baboon) $47.33 \%$ male, $52.67 \%$ female), vaccine group ((baboon) $48.9 \%$ male, $51.1 \%$ female).

\section{Robust humoral immunity induced by vaccination}

Mouse sera from each group were pooled for ELISA. The titres for the total IgG and subtypes were analyzed based on specific time points. In the experimental group, the titres of Sm-p80-specific antibodies were detected starting at week 2 and reached a peak at week 6 , week 8 or week 10 . Among the specific antibodies, the total $\operatorname{IgG}$ and IgG2a remained the highest at 1:204800 and 1:12800 from week 10 and week 12, respectively, and their peaks were delayed 2 weeks in comparison to $\operatorname{IgG} 1, \operatorname{IgG} 2 b$ and $\operatorname{IgA}$. IgG1, IgG2b and IgA reached peaks of 1:6400 at week 8 through week 12 . However, IgG3 failed to exhibit an increase from week 2 onward. IgM started to decrease at week 12 after the peak level of 1:3200 from week 6 to week 10. The peak levels of $\mathrm{rSm}$-p80-specific antibodies in the murine model experiment are shown in figure $1 \mathrm{~A}$.

In baboons from the experimental group, the total $\operatorname{IgG}$ and its subtypes reached a peak of 1:12 800-1:25 600 (IgG), 1:800-1:6400 (IgG1), 1:100-1:400 (IgG2) and 1:200 (IgG3) at week 12; IgM and IgA were at their highest between 1:8001:3200 and 1:1600-1:3200 at week 8, respectively. Figure 1B

Table 2 Adult worm, egg and feces reduction in individual immunized baboon

\begin{tabular}{|c|c|c|c|c|c|c|c|c|c|c|c|}
\hline \multirow[b]{2}{*}{$\begin{array}{l}\text { Vaccine } \\
\text { group }\end{array}$} & \multirow[b]{2}{*}{ Baboon } & \multicolumn{2}{|l|}{ Worm burden } & \multicolumn{3}{|c|}{ Egg burden (per g) } & \multirow[b]{2}{*}{$\begin{array}{l}\text { Total egg burden } \\
\text { in tissue }\end{array}$} & \multicolumn{3}{|c|}{ Egg reduction } & \multirow{2}{*}{$\begin{array}{l}\text { Gross } \\
\text { tissue egg } \\
\text { reduction }\end{array}$} \\
\hline & & Total worm & $\begin{array}{l}\text { Worm } \\
\text { protection }\end{array}$ & Liver & Intestine & Feces & & Liver & Intestine & Feces & \\
\hline \multirow[t]{3}{*}{ GLA-Alum } & Control 1 & 628 & \multirow{3}{*}{$\begin{array}{l}38.53 \% \\
(p=0.005)\end{array}$} & $850.70 \pm 122.92$ & $1985.29 \pm 64.85$ & $96.74 \pm 4.67$ & $1417.99 \pm 261.20$ & \multirow[t]{8}{*}{ No protection } & \multirow[t]{8}{*}{$37.95 \%$} & \multirow[t]{8}{*}{ No protection } & \multirow[t]{8}{*}{$6.81 \%$} \\
\hline & Control 2 & 630 & & $691.68 \pm 64.30$ & $3220.33 \pm 63.28$ & $62.93 \pm 17.78$ & $1956.00 \pm 566.86$ & & & & \\
\hline & Control 3 & 681 & & $2269.70 \pm 32.99$ & $1413.59 \pm 77.00$ & $58.33 \pm 20.90$ & $1841.64 \pm 195.06$ & & & & \\
\hline \multicolumn{2}{|c|}{ Group mean $( \pm \mathrm{SE})$} & $646.33 \pm 17.34$ & & $1270.69 \pm 254.16$ & $2206.40 \pm 268.78$ & $72.67 \pm 9.65$ & $1738.55 \pm 212.30$ & & & & \\
\hline \multirow{3}{*}{$\begin{array}{l}\mathrm{rSm}- \\
\mathrm{p} 80+\mathrm{GLA}- \\
\text { Alum }\end{array}$} & Expt 1 & 319 & & $1290.64 \pm 225.62$ & $2505.46 \pm 207.16$ & $100.93 \pm 15.09$ & $1898.05 \pm 304.23$ & & & & \\
\hline & Expt 2 & 422 & & $1576.43 \pm 89.64$ & $362.90 \pm 67.15$ & $74.50 \pm 4.93$ & $969.66 \pm 275.94$ & & & & \\
\hline & Expt 3 & 451 & & $2747.16 \pm 165.58$ & $1238.88 \pm 73.65$ & $54.63 \pm 24.60$ & $1993.02 \pm 346.86$ & & & & \\
\hline \multicolumn{2}{|c|}{ Group mean $( \pm \mathrm{SE})$} & $397.33 \pm 40.05$ & & $1871.41 \pm 238.39$ & $1369.08 \pm 317.96$ & $76.68 \pm 10.26$ & $1620.24 \pm 202.17$ & & & & \\
\hline
\end{tabular}

GLA-Alum, glucopyranosyl lipid (GLA) formulated in aluminum. 


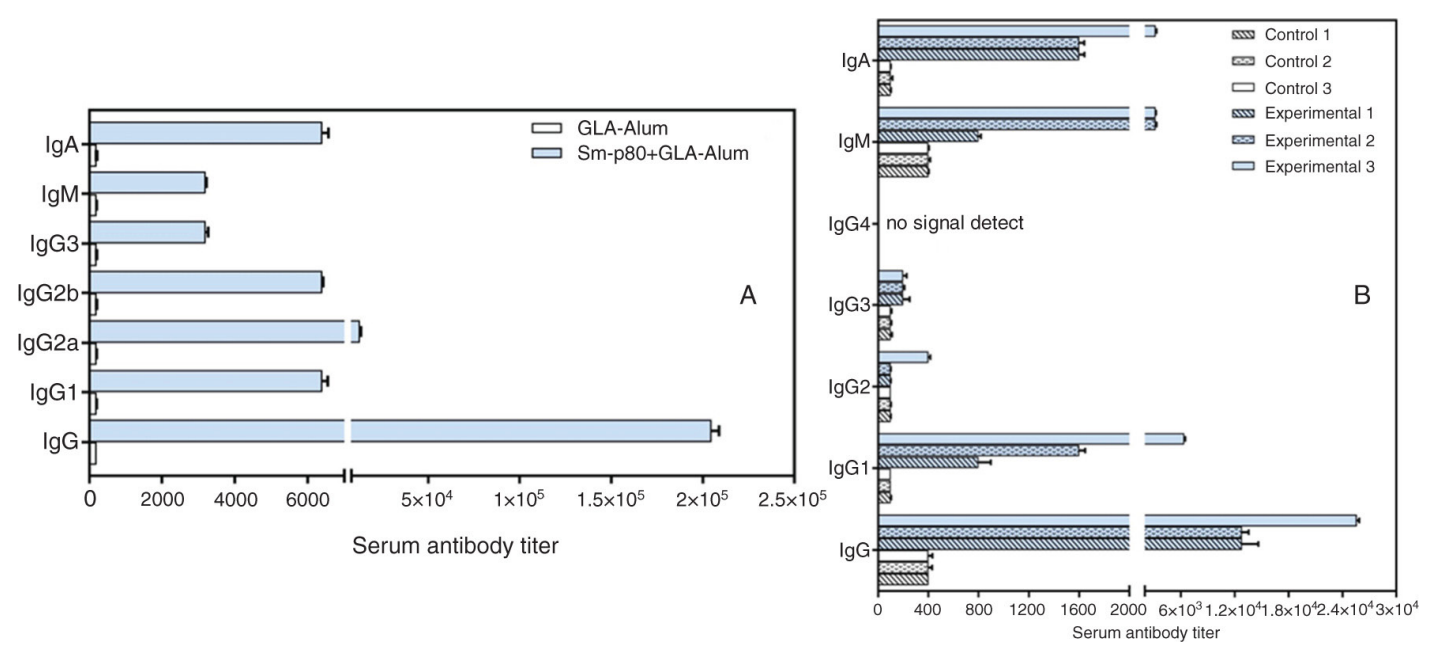

Figure 1 Titres of anti-Sm-p80 antibodies in immunized mice and baboons. ELISAs were performed with sera obtained from each mouse (2-week intervals) and baboon (4-week intervals) in their respective control and experimental groups. Peak antibody titres for total immunoglobulin $(\mathrm{lg}) \mathrm{G}, \lg G 1, \lg \mathrm{G} 2 \mathrm{a}, \lg \mathrm{G} 2 \mathrm{~b}, \lg G 3, \lg \mathrm{M}$ and $\lg A$ in $(\mathrm{A})$ the control and vaccinated mice and (B) the control and vaccinated baboon groups. No signals were detected for IgG4 in either the glucopyranosyl lipid A (GLA) formulated in aluminum (GLA-Alum) control or the $r S m-p 80+G L A-A l u m$ experimental baboons. Values represent the mean of the three experiments $\pm S D$.

shows the peak levels of Sm-p80-specific antibodies from individual baboons following vaccination.

\section{Cell proliferation and cytokine production induced by rSm-p80 re-stimulation}

Specific antigen-induced cell proliferation was tested in vitro. In both experiments, stimulation indices (SI) in ConA-stimulated cells were higher than those stimulated by $\mathrm{rSm}-\mathrm{p} 80$ or OVA (data not shown). Additionally, when comparing the SI from the baboon experimental group (splenocytes: $1.49 \pm 0.07$; PBMC: $1.59 \pm 0.07$; lymph node cells: $1.16 \pm 0.04$ ) with the SI of the control group (splenocytes: 1.25 \pm 0.06 ; PBMC: 1.22 \pm 0.06 ; lymph node cells: $0.96 \pm 0.04)$, cells in the experimental group were more sensitive to rSm-p80 stimulation. After 48 hours incubation with OVA, there were no differences found between the control and experimental groups (data not shown). In the mouse experiment, SI of Sm-p80-stimulated splenocytes between control and experimental groups showed a slight difference, but lacked significance (control: 1.49 \pm 0.33 , experimental: $1.73 \pm 0.77$ ).
The data from cytokine productions in vitro showed a large amount of IFN- $\gamma$ released from all three cell types in experimental groups of baboon experiment and splenocytes in experimental groups of mouse experiment after Sm-p80 restimulation (table 3 ). In addition to IFN- $\gamma$, IL-2 was increasingly synthesized in Sm-p80 specific antigen-triggered cells from experimental groups of mouse and baboon studies. In contrast to IFN- $\gamma$ and IL-2, the production of IL-4 was only increased in mouse splenocytes subsequent to Sm-p80 stimulation, and the increase of IL-10 secretion was only shown in Sm-p80-stimulated splenocytes in experimental groups of mouse and baboon experiments (table 3 ).

\section{mRNA evaluation of cytokines in vitro after rSm-p80 stimulation}

RNA was extracted from PBMCs, lymph node cells and splenocytes from baboons and from splenocytes of mice following incubation with rSm-p80. mRNA expression of 23 cytokines in the mice and of 18 cytokines in baboons was estimated via RT-PCR. The profiles of mRNA

\begin{tabular}{|c|c|c|c|c|c|c|}
\hline & \multirow[b]{2}{*}{ Cell } & \multirow[b]{2}{*}{ Vaccine group } & \multicolumn{4}{|c|}{ Cytokine concentration in $\mathrm{pg} / \mathrm{mL}($ mean $\mathrm{SD})$} \\
\hline & & & IL-2 & IL-4 & IL-10 & IFN- $\gamma$ \\
\hline \multirow[t]{6}{*}{ Baboon } & PBMCs & GLA-Alum & $55.26 \pm 7.05$ & $37.68 \pm 1.16$ & $164.10 \pm 44.94$ & $132.64 \pm 34.11$ \\
\hline & & rSm-p80+GLA-Alum & $608.28 \pm 399.24^{*}$ & $38.04 \pm 0.91$ & $268.70 \pm 91.00$ & $530.58 \pm 103.49^{*}$ \\
\hline & Lymph node cells & GLA-Alum & $66.54 \pm 4.83$ & $48.27 \pm 2.86$ & $71.18 \pm 5.15$ & $64.44 \pm 3.40$ \\
\hline & & rSm-p80+GLA-Alum & $537.78 \pm 28.20^{*}$ & $61.55 \pm 19.41$ & $67.17 \pm 7.54$ & $191.34 \pm 3.73^{*}$ \\
\hline & Splenocytes & GLA-Alum & $36.71 \pm 1.28$ & $30.68 \pm 1.75$ & $34.13 \pm 1.03$ & $86.29 \pm 16.31$ \\
\hline & & rSm-p80+GLA-Alum & $120.15 \pm 8.11^{*}$ & $35.55 \pm 1.55$ & $157.71 \pm 63.97^{*}$ & $359.71 \pm 96.51^{*}$ \\
\hline \multirow[t]{2}{*}{ Mice } & Splenocytes & GLA-Alum & $30.80 \pm 1.55$ & $259.38 \pm 16.31$ & $477.34 \pm 9.06$ & $779.92 \pm 16.59$ \\
\hline & & rSm-p80+GLA-Alum & $902.11 \pm 8.02^{*}$ & $706.36 \pm 13.06^{*}$ & $634.44 \pm 39.27^{*}$ & $1038.32 \pm 47.41^{*}$ \\
\hline
\end{tabular}

${ }^{*} P \leq 0.05$ versus respective control groups stimulated by recombinant $S m-p 80$ using independent samples test.

GLA-Alum, glucopyranosyl lipid A (GLA) formulated in aluminum (GLA-Alum); IFN, interferon; IL, interleukin; PBMC, peripheral blood mononuclear cells. 

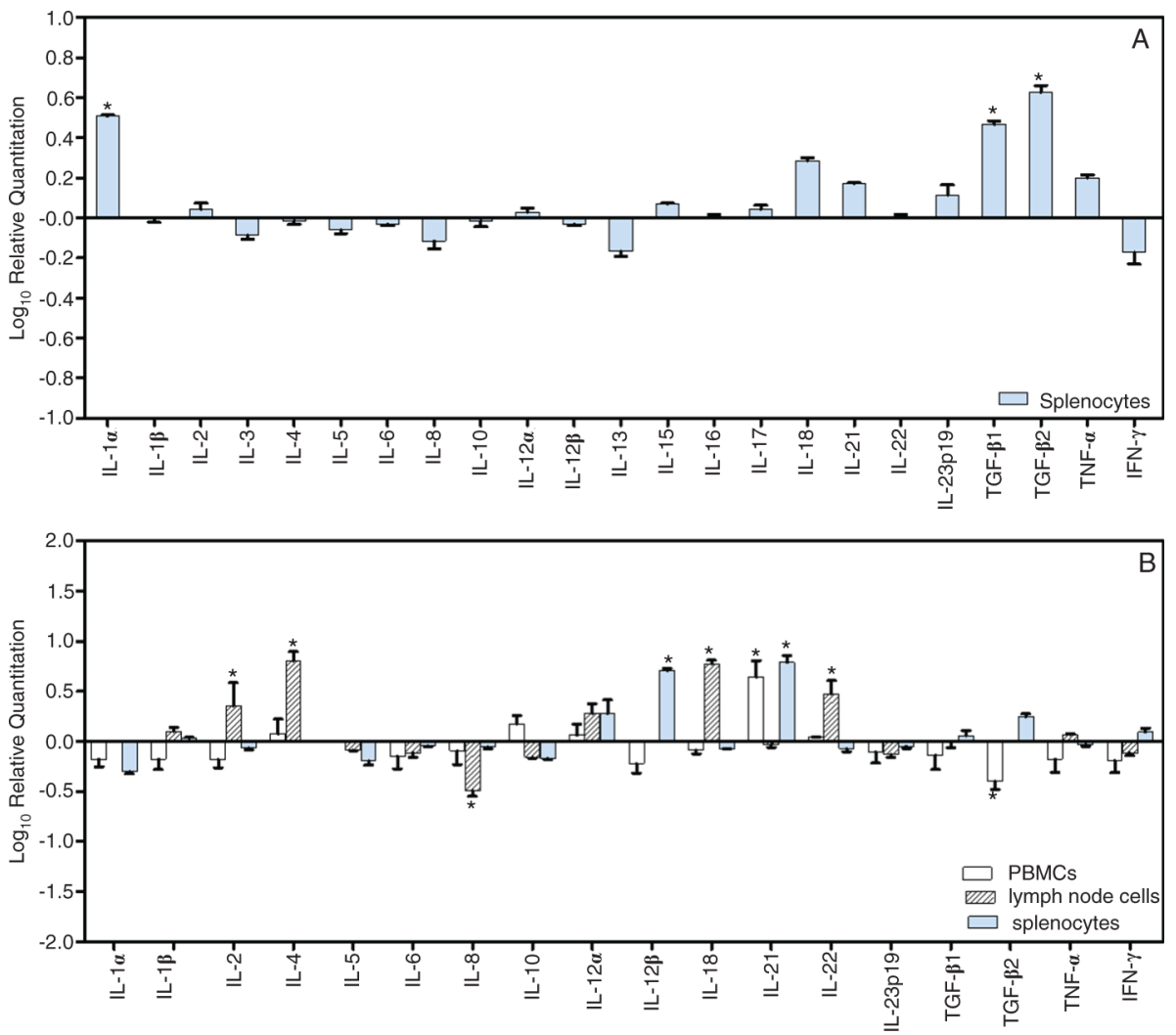

Figure 2 Relative fold changes of cytokine mRNA expression in vaccinated mice and baboons. After 24 hours incubation with rSm-p80, RNA was extracted from stimulated pooled peripheral blood mononuclear cells (PBMCs), lymph node cells and splenocytes attained from baboons and from stimulated pooled splenocytes isolated from mice. After standardization using glyceraldehyde 3-phosphate dehydrogenase (GAPDH) through Quantity One Programme software V.4.6.2, the relative ratio of cytokine mRNA expression was compared between the control and experimental group. Panel A showed the expression of 23 cytokines in vaccinated mice and panel B showed the relative expression of 18 cytokines in vaccinated baboons.

expression are summarized in figure $2 \mathrm{~A}, \mathrm{~B}$. In the mouse experimental group, following 24 hours of rSm-p80 stimulation, major variations only occurred in inflammatory cytokines (IL-1 $\alpha$ and IL-18) and the TGF family. Others showed no significant changes. In the baboon experimental group, upregulation of IL-21 was observed in PBMCs. Some Th1 (IL-2 and IL-18), Th2 (IL-4) and Th17
(IL-22 or IL-21) cytokines were modulated by a specific rSm-p80 antigen in lymph node cells and splenocytes.

\section{Recombinant Sm-p80 stimulated SFU determined by ELISPOT}

In the mouse experiment, a mean of 15.5 IFN- $\gamma$ SFU per million splenocytes was detected in the control group and
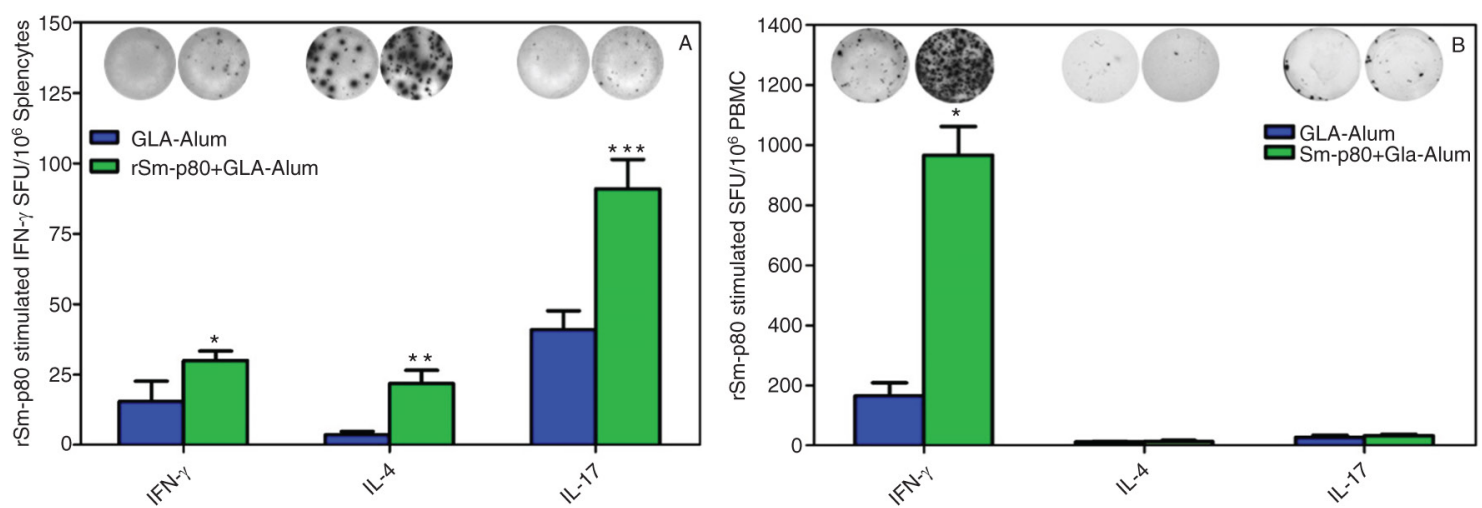

Figure 3 Detection of Th1/Th2/Th17 secreting cells in splenocytes of mice (A) and peripheral blood mononuclear cells (PBMCs) of baboons (B). Interferon (IFN)- $\gamma$, interleukin (IL)-4 and IL-17 are typical cytokine markers for Th1/Th2/Th17 subsets. IFN- $\gamma$, IL-4 and IL-17 secreting cells were assayed by Enzyme-Linked ImmunoSpot. Spot-forming units (SFUs) were calculated in one million cells. The SFUs for cells obtained from the control group (glucopyranosyl lipid A (GLA) formulated in aluminum (GLA-Alum)) are shown as blue bars, and green bars represent $\mathrm{rSm}-\mathrm{p} 80$ stimulated cells from the experimental group ( $\mathrm{Sm}-\mathrm{p} 80+\mathrm{GLA}-\mathrm{Alum}$ ). 
30 SFU per million formed in the experimental group. This showed an approximate twofold increase between the control and experimental group. The number of IL-4 SFU per million showed a 6.2-fold increase in the experimental group compared with the control group. For IL-17, 41 SFU per million were observed in control group and 91 SFU per million were observed in experimental group, exhibiting a 2.2-fold increase compared with the control group (figure $3 \mathrm{~A}$ ). In the baboon experiment, the mean of the control group was 165 IFN- $\gamma$ secreting SFUs per million PBMCs and 967 IFN- $\gamma$ secreting SFUs per million PBMCs in the experimental group. This indicated more than a 5.9-fold increase in IFN- $\gamma$ secreting SFU per million in rSm-p80 formulated in GLA-Alum vaccinated animals when compared with their respective control group (adjuvant alone). Although the difference was seen in IFN- $\gamma$ secreting SFU per million in PBMCs, there was no significance related to IL-4 and IL-17 secreting
PBMCs in either the control or the experimental group (figure 3B).

\section{Th1, Th2 and Th17 subset assay via fluorescence- activated cell sorting}

Flow cytometry was performed to determine the roles of Th1, Th2 and Th17 responses in Sm-p80-mediated protection. Comparison of both control and experimental mouse groups showed no significant difference between the percentages of IFN- $\gamma$ and IL-17 secreting splenocytes (figure 4A). However, the percentage of IL-4 secreting splenocytes in the experimental group had a 2.4-fold increase in comparison to the control group. Similarly, the percentages of IFN- $\gamma$ and IL-17 secreting PBMCs in the baboon experiment did not exhibit a noticeable difference between the control and experimental group. However, the percentage of IL-4 secreting PBMCs in the experimental group

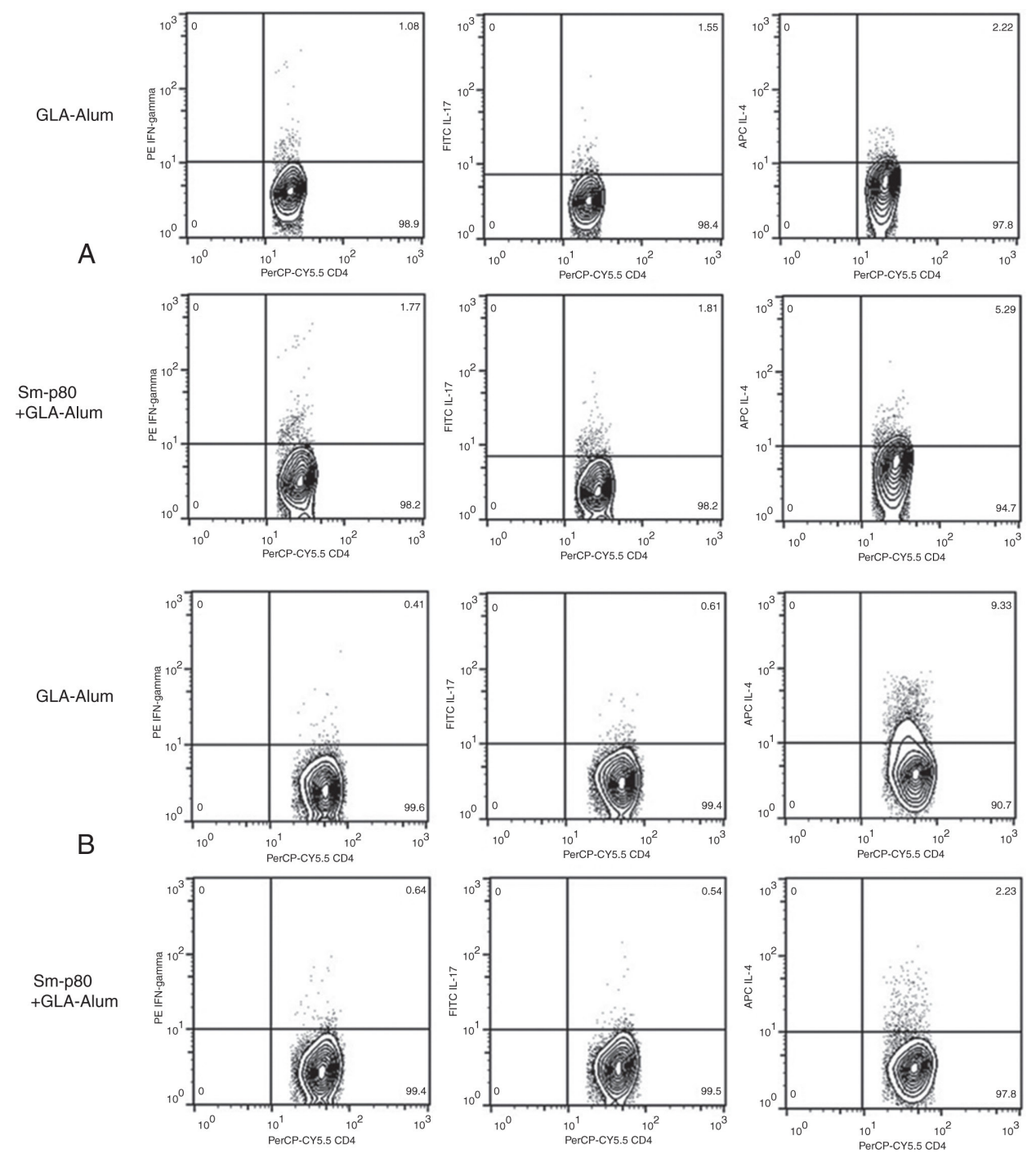

Figure 4 Quantification of intracellular interferon (IFN)- $\gamma$, interleukin (IL)-4 and IL-17 secretory CD3+CD4+T cells from rSm-p80 stimulated splenocytes from the mouse experiment and peripheral blood mononuclear cells from the baboon experiment. Initial gating

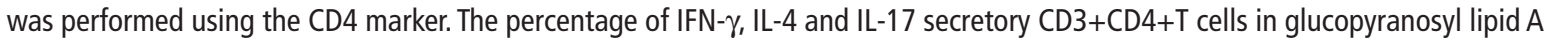
(GLA) formulated in aluminum (GLA-Alum) and rSm-p80 formulated in GLA-Alum vaccinated groups were shown in mice (panel A) and baboons (panel B). 
was reduced by $7 \%$ in comparison to the control group (figure 4B).

\section{DISCUSSION}

Over the past two decades, our group has adopted a systematic approach to develop a Sm-p80-based schistosomiasis vaccine. Efficacy studies using different $\mathrm{Sm}$-p80 vaccine formulations and/or strategies in rodent and baboon models of infection and disease have consistently shown promising results. ${ }^{61012-161925-34}$ In this present study, we assessed the efficacy of Sm-p80 antigen combined with GLA adsorbed on aluminum hydroxide (Sm-p80+GLA-Alum) in mice and in a non-human primate pilot study. Our results showed that vaccination with Sm-p80+GLA-Alum reduced worm burden by $39 \%-44 \%$ and intestinal egg load by $38 \%$ in immunized mice and baboons, but not as significant as we have previously published with either Sm-p80+GLA-SE or Sm-p80+Alumalone ${ }^{16}$ formulations. This result indicates that GLA-Alum adjuvant does not provide improvement in Sm-p 80 vaccine efficacy despite the successes reported in other systems using GLA-Alum such as ID93-based vaccine against tuberculosis. ${ }^{35}$

High antibody production plays a significant role in immune protection against schistosomiasis. ${ }^{27} 29$ 36-38 For instance, studies utilizing natural host of schistosomes (rhesus macaques) and/or semi-permissive hosts (outbred rats) have shown that resistance to schistosome infections are almost entirely antibody mediated. ${ }^{38-40}$ Immunizations with Sm-p80+GLA-Alum induced robust production of Sm-p80-specific total $\operatorname{IgG}$ and $\operatorname{IgG}$ subtypes (IgG1, IgG2, $\operatorname{IgG} 2$ and $\operatorname{IgG} 3$ ) in both mice and baboons in addition to significant IgA and IgM production in vaccinated baboons.

The importance of Th1 type immune responses in the elimination of schistosomiasis has been demonstrated by our group and others. ${ }^{10} 36374142$ Studies with radiation attenuated (RA) and recombinant vaccines in mouse and baboon models in addition to data from individuals naturally resistant to schistosomiasis (endemic normals or putative resistant individuals) ${ }^{43}$ further support the role of strong Th1 immune responses (IFN- $\gamma$, IL-2, IL- $1 \alpha$ and TNF- $\alpha$ ) in immune-mediated protection against schistosome infections. ${ }^{12} 163744$ In this study, we observed an increased production of Th1 cytokines (IFN- $\gamma$, IL- 2 and TNF- $\alpha$ ) by circulating immune cells in animals immunized with Sm-p80+GLA-Alum. Interestingly, the production of intracellular Th2 and Th17 cytokines in stimulated splenocytes was only significant in vaccinated mice and not in the baboons. However, there was significant induction of Th2 cytokines (IL-4 and IL-1 $\beta$ ) in stimulated lymph node cells from vaccinated baboons when compared with the control animals. Overall, Sm-p80+GLA-Alum elicited a mixed Th1/ Th2/Th17 immune responses which appeared to correlate with the protection observed. A mixed Th1/Th2 type immune responses have been associated with protection against schistosomiasis with Th2 responses thought to play significant roles. ${ }^{45}$ The induction of Th2-type responses is known to contribute to the recruitment of basophils and eosinophils which are thought to be involved in schistosomula killing in vivo. ${ }^{46}$

In summary, the data presented in this current study demonstrated that $S \mathrm{~m}-\mathrm{p} 80$ vaccine formulated in GLA-Alum offered a moderate but significant protection against $S$. mansoni infections in both mice and baboons. However, our findings show that despite the diverse immune responses induced by Sm-p80+GLA-Alum formulation, we submit that GLA-Alum is insufficient in maximizing the immunogenicity of the Sm-p80 antigen. Therefore, GLA-Alum may not be suitable for the next of our Sm-p80 vaccine development.

\section{Author affiliations}

${ }^{1}$ Center for Tropical Medicine and Infectious Diseases, Texas Tech University Health Sciences Center, Lubbock, Texas, USA

${ }^{2}$ Department of Internal Medicine, Texas Tech University Health Sciences Center, Lubbock, Texas, USA

${ }^{3}$ Department of Natural Sciences, School of Arts \& Sciences, Peru State College, Peru, Nebraska, USA

${ }^{4}$ Comparative Pathology/lmmunology, Tulane National Primate Research Center, Covington, Louisiana, USA

${ }^{5}$ Bladder Immunology Group, Biomedical Research Institute, Rockville, Maryland, USA

${ }^{6}$ PAI Life Sciences, Seattle, Washington, USA

Acknowledgements We thank Dr Steve Reed for providing GLA-Alum adjuvant for these studies. We would also like to thank Whitni Redman for critically reviewing the manuscript.

Contributors WZ, GA, WT, AJM, LL and AJS performed the experiments. WZ, AJM, WT, EK and SL wrote the manuscript and performed the statistical analysis. AAS designed the experimental plan.

Funding This work was supported in part by grants from the Bill and Melinda Gates Foundation grant (OPP1097535) and from the NIAID/NIH SBIR (R43/R44 Al103983). The snails were supplied through a NIH-NIAID contract (HHSN2722010000051) to Biomedical Research Institute.

Competing interests None declared.

Patient consent Not required.

Ethics approval Institutional Animal Care and Use Committee.

Provenance and peer review Not commissioned; externally peer reviewed.

Open access This is an open access article distributed in accordance with the Creative Commons Attribution Non Commercial (CC BY-NC 4.0) license, which permits others to distribute, remix, adapt, build upon this work noncommercially, and license their derivative works on different terms, provided the original work is properly cited and the use is non-commercial. See: http:// creativecommons.org/licenses/by-nc/4.0/

(C) American Federation for Medical Research (unless otherwise stated in the text of the article) 2018. All rights reserved. No commercial use is permitted unless otherwise expressly granted.

\section{REFERENCES}

1 Chitsulo L, Loverde P, Engels D. Schistosomiasis. Nat Rev Microbiol 2004;2:12-13.

2 Gryseels B, Polman K, Clerinx J, et al. Human schistosomiasis. Lancet 2006;368:1106-18.

3 Colley DG, Bustinduy AL, Secor WE, et al. Human schistosomiasis. Lancet 2014;383:2253-64.

4 Mo AX, Agosti JM, Walson JL, et al. Schistosomiasis elimination strategies and potential role of a vaccine in achieving global health goals. Am J Trop Med Hyg 2014:90:54-60.

5 Terer CC, Bustinduy AL, Magtanong RV, et al. Evaluation of the health-related quality of life of children in Schistosoma haematobium-endemic communities in Kenya: a cross-sectional study. PLoS Negl Trop Dis 2013;7:e2106.

6 Siddiqui AA, Siddiqui SZ. Sm-p80-based schistosomiasis vaccine: preparation for human clinical trials. Trends Parasitol 2017;33:194-201.

7 Mo AX, Colley DG. Workshop report: Schistosomiasis vaccine clinical development and product characteristics. Vaccine 2016;34:995-1001.

8 Andresen K, Tom TD, Strand M. Characterization of CDNA clones encoding a novel calcium-activated neutral proteinase from Schistosoma mansoni. J Biol Chem 1991;266:15085-90.

9 Young BW, Podesta RB. Complement and 5-HT increase phosphatidylcholine incorporation into the outer bilayers of Schistosoma mansoni. J Parasitol $1986 ; 72: 802-3$. 
10 Molehin AJ, Sennoune SR, Zhang W, et al. Cross-species prophylactic efficacy of Sm-p80-based vaccine and intracellular localization of Sm-p80/Sm-p80 ortholog proteins during development in Schistosoma mansoni, Schistosoma japonicum, and Schistosoma haematobium. Parasitol Res 2017;116:3175-88.

11 Siddiqui AA, Zhou Y, Podesta RB, et al. Characterization of $\mathrm{Ca}(2+)$-dependent neutral protease (calpain) from human blood flukes, Schistosoma mansoni. Biochim Biophys Acta 1993;1181:37-44.

12 Ahmad G, Zhang W, Torben W, et al. Preclinical prophylactic efficacy testing of Sm-p80-based vaccine in a nonhuman primate model of Schistosoma mansoni infection and immunoglobulin $\mathrm{G}$ and $\mathrm{E}$ responses to $\mathrm{Sm}$-p 80 in human serum samples from an area where schistosomiasis is endemic. J Infect Dis 2011;204:1437-49.

13 Ahmad G, Zhang W, Torben W, et al. Protective and antifecundity effects of Sm-p80-based DNA vaccine formulation against Schistosoma mansoni in a nonhuman primate model. Vaccine 2009;27:2830-7.

14 Karmakar S, Zhang W, Ahmad G, et al. Cross-species protection: Schistosoma mansoni Sm-p80 vaccine confers protection against Schistosoma haematobium in hamsters and baboons. Vaccine 2014;32:1296-303.

15 Zhang W, Ahmad G, Torben W, et al. Sm-p80-based DNA vaccine made in a human use approved vector VR1020 protects against challenge infection with Schistosoma mansoni in mouse. Parasite Immunol 2010;32:252-8.

16 Zhang W, Ahmad G, Torben W, et al. Schistosoma mansoni antigen Sm-p80: Prophylactic efficacy of a vaccine formulated in human approved plasmid vector and adjuvant (VR 1020 and alum). Acta Trop 2011;118:142-51.

17 Guy B. The perfect mix: recent progress in adjuvant research. Nat Rev Microbiol 2007;5:505-17.

18 Warshakoon HJ, Hood JD, Kimbrell MR, et al. Potential adjuvantic properties of innate immune stimuli. Hum Vaccin 2009;5:381-94.

19 Karmakar S, Zhang W, Ahmad G, et al. Use of an Sm-p80-based therapeutic vaccine to kill established adult schistosome parasites in chronically infected baboons. J Infect Dis 2014;209:1929-40.

20 Coler RN, Bertholet S, Moutaftsi M, et al. Development and characterization of synthetic glucopyranosyl lipid adjuvant system as a vaccine adjuvant. PLoS One 2011;6:e16333.

21 Lindblad EB. Aluminium compounds for use in vaccines. Immunol Cell Biol 2004;82:497-505.

22 Damian RT, Greene ND, Fitzgerald K. Schistosomiasis mansoni in baboons. The effect of surgical transfer of adult Schistosoma mansoni upon subsequent challenge infection. Am J Trop Med Hyg 1972;21:951-8.

23 Tucker MS, Karunaratne LB, Lewis FA, et al. Schistosomiasis. Curr Protoc Immunol 2013:103.

24 Frey A, Di Canzio J, Zurakowski D. A statistically defined endpoint titer determination method for immunoassays. J Immunol Methods 1998;221:35-41.

25 Ahmad G, Torben W, Zhang W, et al. Sm-p80-based DNA vaccine formulation induces potent protective immunity against Schistosoma mansoni. Parasite Immunol 2009;31:156-61.

26 Ahmad G, Zhang W, Torben W, et al. Prime-boost and recombinant protein vaccination strategies using $\mathrm{Sm}$-p 80 protects against Schistosoma mansoni infection in the mouse model to levels previously attainable only by the irradiated cercarial vaccine. Parasitol Res 2009;105:1767-77.

27 Zhang W, Ahmad G, Le L, et al. Longevity of Sm-p80-specific antibody responses following vaccination with $\mathrm{Sm}-\mathrm{p} 80$ vaccine in mice and baboons and transplacental transfer of Sm-p80-specific antibodies in a baboon. Parasitol Res 2014;113:2239-50.

28 Zhang W, Ahmad G, Torben W, et al. Sm-p80-based DNA vaccine provides baboons with levels of protection against Schistosoma mansoni infection comparable to those achieved by the irradiated cercarial vaccine. J Infect Dis 2010;201:1105-12.

29 Torben W, Ahmad G, Zhang W, et al. Role of antibodies in Sm-p80-mediated protection against Schistosoma mansoni challenge infection in murine and nonhuman primate models. Vaccine 2011;29:2262-71.

30 Ahmad G, Zhang W, Torben W, et al. Protective effects of Sm-p80 in the presence of resiquimod as an adjuvant against challenge infection with Schistosoma mansoni in mice. Int J Infect Dis 2010;14:e781-7.

31 Le L, Zhang W, Karmakar S, et al. Simultaneous priming with DNA encoding Sm-p80 and boosting with Sm-p80 protein confers protection against challenge infection with Schistosoma mansoni in mice. Parasitol Res 2014;113:1195-200.

32 Siddiqui $A A$, Phillips T, Charest $H$, et al. Induction of protective immunity against Schistosoma mansoni via DNA priming and boosting with the large subunit of calpain (Sm-p80): adjuvant effects of granulocytemacrophage colony-stimulating factor and interleukin-4. Infect Immun 2003; 71:3844-51.

33 Siddiqui AA, Phillips T, Charest H, et al. Enhancement of Sm-p80 (large subunit of calpain) induced protective immunity against Schistosoma mansoni through co-delivery of interleukin-2 and interleukin-12 in a DNA vaccine formulation. Vaccine 2003;21:2882-9.

34 Siddiqui AA, Pinkston JR, Quinlin ML, et al. Characterization of protective immunity induced against Schistosoma mansoni via DNA priming with the large subunit of calpain (Sm-p80) in the presence of genetic adjuvants. Parasite 2005;12:3-8.

35 Orr MT, Fox CB, Baldwin SL, et al. Adjuvant formulation structure and composition are critical for the development of an effective vaccine against tuberculosis. J Control Release 2013;172:190-200.

36 Wilson RA, Coulson PS. Immune effector mechanisms against schistosomiasis: looking for a chink in the parasite's armour. Trends Parasitol 2009;25:423-31.

37 Wilson RA, Coulson PS, Mountford AP. Immune responses to the radiationattenuated schistosome vaccine: what can we learn from knock-out mice? Immunol Lett 1999;65:117-23.

38 Wilson RA, Langermans JA, van Dam GJ, et al. Elimination of Schistosoma mansoni adult worms by Rhesus Macaques: basis for a therapeutic vaccine? PLoS Negl Trop Dis 2008;2:e290.

39 Knopf PM, Nutman TB, Reasoner JA. Schistosoma mansoni: resistance to reinfection in the rat. Exp Parasitol 1977;41:74-82.

40 Li XH, Xu YX, Vance G, et al. Evidence that Rhesus macaques self-cure from a Schistosoma japonicum infection by disrupting worm esophageal function: a new route to an effective vaccine? PLoS Negl Trop Dis 2015;9:e0003925.

41 Ricciardi A, Visitsunthorn K, Dalton JP, et al. A vaccine consisting of Schistosoma mansoni cathepsin B formulated in Montanide ISA 720 VG induces high level protection against murine schistosomiasis. BMC Infect Dis 2016;16:112.

42 Wilson RA, Li XH, Castro-Borges W. Do schistosome vaccine trials in mice have an intrinsic flaw that generates spurious protection data? Parasit Vectors 2016;9:89.

43 Corrêa-Oliveira R, Caldas IR, Gazzinelli G. Natural versus drug-induced resistance in Schistosoma mansoni infection. Parasitol Today 2000;16:397-9.

44 McManus DP, Loukas A. Current status of vaccines for schistosomiasis. Clin Microbiol Rev 2008;21:225-42.

45 Pearce EJ, MacDonald AS. The immunobiology of schistosomiasis. Nat Rev Immunol 2002;2:499-511.

46 El Ridi R, Tallima H. Vaccine-induced protection against murine schistosomiasis mansoni with larval excretory-secretory antigens and papain or type-2 cytokines. J Parasitol 2013;99:194-202. 